Check for updates

Cite this: RSC Adv., 2017, 7, 39530

\title{
Adsorption mechanism of mussel-derived adhesive proteins onto various self-assembled monolayers $\uparrow$
}

\author{
Shizhe Huang, ${ }^{\text {ab }}$ Qingfeng Hou, ${ }^{c}$ Donghong Guo, ${ }^{c}$ Hui Yang, ${ }^{\text {a }}$ Ting Chen, ${ }^{\text {ab }}$ \\ Fanghui Liu, ${ }^{a}$ Guangxin $\mathrm{Hu}^{a}{ }^{a}$ Minghui Zhang, ${ }^{a}$ Jian Zhang ${ }^{d}$ and Jinben Wang (D) *a
}

Mussel adhesion to a variety of surfaces has received considerable attention due to its ability to bind strongly to many surfaces under water. Understanding the interactions between mussel-derived adhesive proteins and surfaces with different chemical and physical properties is of great theoretical and practical interest. Here, we explored the adsorption behavior of mussel foot protein-1 (Mfp-1) onto self-assembled monolayers (SAMs) with varying wettability and chemistry, through quartz crystal microbalance with dissipation measurements, ellipsometry, X-ray photoelectron spectroscopy, and atomic force microscopy. The results showed significant differences in the structural conformations of protein adsorbed layers for the series of surfaces. Two mechanisms were found in all the systems; in the case of hydrophobic surfaces, the first regime corresponded to the initial adsorption of protein molecules onto the surfaces, and the second kinetic process was related to conformational changes, resulting in a relatively rigid and dense protein layer; while for hydrophilic surfaces, a loose and soft adsorbed protein film was generated. It was found that the adsorbed mass of Mfp-1 at the hydroxyl terminated SAM surface was the smallest among all the modified surfaces, because of the formation of hydration layers reducing protein adhesion effectively. Furthermore, the interaction mechanisms of protein molecules with solid surfaces were suggested, providing a new way of designing and developing underwater adhesive or anti-fouling materials.

Received 5th July 2017

Accepted 6th August 2017

DOI: 10.1039/c7ra07425e

rsc.li/rsc-advances the adhesive strategies of the adhesive protein have aroused broad interest both in theoretical and technological fields. ${ }^{9-15}$

Current studies have investigated the wet adhesion of Mfp-1 to different surfaces, such as mica or metal oxide, while in natural world, surfaces are covered by various organic films within minutes. ${ }^{16,17}$ So, it is necessary to understand mussel binding mechanisms to such fouled films due to the great importance in marine biofouling and the biocompatibility of biomaterials. Besides, there is currently a lack of knowledge about how material factors, such as surface chemistry, can control protein adhesion/morphology. ${ }^{18}$ There are only a few pioneering works reported on how material properties can affect protein adsorption, ${ }^{19-21}$ e.g., methyl and hydroxyl terminated self-assembled monolayer (SAM) surfaces were prepared to create hydrophobic and hydrophilic surfaces, respectively, and reduce the protein adsorption to some extent; however, little is understood about how changing intricate surface chemistry can influence Mfp-1 adsorption and adhesion.

In order to address these questions, we have prepared a series of model surfaces modified by SAMs varying systematically in water wettability, from hydrophilic to hydrophobic, including hydroxyl $(-\mathrm{OH})$, amino $\left(-\mathrm{NH}_{2}\right)$, methyl $\left(-\mathrm{CH}_{3}\right)$, and perfluorodecane $\left(-\mathrm{CF}_{3}\right)$ terminated groups in the present work. The first three functional groups can be found naturally within biological systems and the last one contributes low surface
${ }^{a}$ Key Laboratory of Colloid, Interface and Chemical Thermodynamics, Institute of

University of Chinese Academy of Sciences, Beijing 100049, P. R. China

${ }^{c}$ Key Laboratory of Oilfield Chemistry, Research Institute of Petroleum Exploration and Development (RIPED), CNPC, Beijing 100083, P. R. China

${ }^{d}$ CNOOC Research Institute, State Key Laboratory of Offshore Oil Exploitation, Beijing 100027, People's Republic of China

$\dagger$ Electronic supplementary information (ESI) available. See DOI: 10.1039/c7ra07425e 
energy, using thiol modification techniques on gold surface. The interactions between wet surfaces in the presence of Mfps adsorbed layers were normally studied using surface forces apparatus (SFA), ${ }^{22-24}$ but such measurement can hardly provide the information about adsorption of proteins on the solid/water interface and the displacement of water molecules from the interface in situ which is the first step to understanding adhesive mechanisms. In this work, detailed information about the adsorption and film-forming processes of Mfp-1 on such surfaces has been determined by quartz crystal microbalance with dissipation monitoring (QCM-D), ellipsometry, X-ray photoelectron spectroscopy (XPS), and atomic force microscopy (AFM) measurements. The effects of chemistry composition and water wettability of SAMs on the adsorption process of Mfp- 1 have been studied, leading to a better understanding to the interactions between protein molecules or aggregates or between protein and surface.

\section{Materials and methods}

\section{Materials}

The Mfp-1 (purity 90\%, Sigma-Aldrich) was diluted to $0.01 \mathrm{mg} \mathrm{mL}^{-1}$ in $0.1 \mathrm{M}$ citric acid buffer ( $\mathrm{pH}$ 5.5). The $\mathrm{pH}$ of this buffer is below the upper limit at which Mfp-1 undergoes spontaneous oxidation and subsequent cross-linking/ aggregation in solution. ${ }^{25,26}$ Citric acid buffer was prepared by first dissolving the required quantity of citric acid (AR, purity 99.5\%, Sigma-Aldrich) in Millipore MilliQ water, and then adding small amounts of sodium hydroxide (AR, purity 98\%, Sigma-Aldrich). 1-Octadecanethiol (AR, purity 96\%, J\&K Scientific), 11-mercapto-1-undecane (AR, purity 96\%, J\&K Scientific), $1 H, 1 H, 2 H, 2 H$-perfluorodecane-1-thiol (AR, purity $97 \%$, J\&K Scientific), and 11-amino-1-undecanethiol hydrochloride (AR, purity 99\%, Sigma-Aldrich) were used for the synthesis of selfassembled monolayers (SAMs), respectively.

\section{Preparation and characterization of self-assembled monolayers (SAMs)}

Before preparing SAMs, QCM gold sensor chips were immersed in piranha solution $(3: 1 \mathrm{vol}$. ratio of concentrated sulfuric acid and $30 \% \mathrm{H}_{2} \mathrm{O}_{2}$ ) at $75{ }^{\circ} \mathrm{C}$ for $15 \mathrm{~min}$, and then rinsed with deionized water and dried with pure nitrogen. To create SAMfunctionalized substrates, the freshly clean chips were immersed in ethanolic solutions of 1-octadecanethiol, 11mercapto-1-undecane, $1 \mathrm{H}, 1 \mathrm{H}, 2 \mathrm{H}, 2 \mathrm{H}$-perfluorodecane-1-thiol, and 11-amino-1-undecanethiol hydrochloride at the concentration of $1 \mathrm{mM}$ for $24 \mathrm{~h}$, respectively. ${ }^{27}$ The substrates were thoroughly rinsed by ethanol to remove physisorbed thiol from the surfaces and dried with pure nitrogen stream.

The wetting of aqueous drops on the SAM-modified surfaces were determined through a contact angle goniometer with an OCA20 system (DataPhysics, Germany) at room temperature. The elemental surface component of SAMs was determined by X-ray photoelectron spectroscopy (XPS) using ESCALab 250Xi with $200 \mathrm{~W}$ monochromated $\mathrm{Al} \mathrm{K} \alpha$ radiation. The base pressure in the analysis chamber was about $3 \times 10^{-10} \mathrm{mbar}$. Typically the hydrocarbon $\mathrm{C} 1 \mathrm{~s}$ line at $284.8 \mathrm{eV}$ from adventitious carbon is used for energy referencing.

\section{Quartz crystal microbalance with dissipation monitoring (QCM-D)}

The protein adsorption mass and characteristics of the adsorbed layer were assessed using a quartz crystal microbalance with dissipation (QCM-D) from Biolin Scientific AB (Q-sense E1, Sweden). A sensor of gold-coated quartz crystal with AT-cut (QSX 301, Biolin Scientific AB, Sweden) was used, with a fundamental resonant frequency of $5 \mathrm{MHz}$ and a mass sensitivity constant $(C)$ of $17.7 \mathrm{ng} \mathrm{cm}^{-2} \mathrm{~Hz}^{-1}$. The SAM-modified surface was exposed to the protein solution. When a quartz crystal is excited to oscillate in the thickness shear mode at its fundamental resonance frequency $\left(f_{0}\right)$ by applying a RF voltage across the electrodes near the resonance frequency, an adsorption layer added to the electrodes induces a decrease in resonance frequency $(\Delta f)$. The QCM-D technique monitors the frequency and energy dissipation of the oscillating shear motion of a quartz crystal at all the harmonics $(n=1,3,5, \ldots, 13)$, which provides the information about the mass of adsorbate on the surface and the elastic property of the adsorbed film. The uncertainty is below

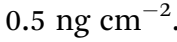

For a rigid and thin layer, adsorption curves at different overtones are similar, so the adsorbed mass on the sensor is generally obtained through the Sauerbrey equation: ${ }^{28}$

$$
\Delta m=-\frac{\rho_{\mathrm{q}} l_{\mathrm{q}}}{f_{0}} \frac{\Delta f}{n}=-C \frac{\Delta f}{n}
$$

where $f_{0}$ is the fundamental frequency; $\rho_{\mathrm{q}}$ and $l_{\mathrm{q}}$ are the specific density and thickness of the quartz crystal, respectively. The dissipation factor is defined by eqn (2): ${ }^{29}$

$$
\Delta D=\frac{E_{\mathrm{d}}}{2 \pi E_{\mathrm{s}}}
$$

where $E_{\mathrm{d}}$ and $E_{\mathrm{s}}$ are the dissipated and stored energy during one oscillation, respectively. The third overtone $(n=3)$ is used when QCM data are processed through the Sauerbrey equation.

For a viscoelastic layer, the layer is not fully coupled to the oscillation of the crystal and undergoes a deformation under shear oscillatory motion, in which the crystal's oscillation is dampened and the change in resonant frequency is influenced. So the Sauerbrey equation is not valid and it is necessary to use the Voigt model. In such calculations, the parameters of density and viscosity of protein solutions were used as the values of $1002 \mathrm{~kg} \mathrm{~m}^{-3}$ and $0.00103 \mathrm{~Pa} \mathrm{~s}$ at room temperature, respectively. ${ }^{30}$ The best fitting values of the shear viscosity $(\eta)$, shear modulus $(\mu)$, and thickness $(h)$ of the adsorbed layer were obtained by modeling the experimental data of $f$ and $D$ for three overtones using the Q-tools software package (Biolin Scientific $\mathrm{AB}$, Sweden).

All QCM-D experiments were conducted in a flow-through mode at a rate of $100 \mu \mathrm{L} \min ^{-1}$ at $\sim 25{ }^{\circ} \mathrm{C}$. A baseline was established by citric acid buffer for at least $5 \mathrm{~min}$. After the Mfp1 solution ( $0.1 \mathrm{mg} \mathrm{mL} \mathrm{m}^{-1}$ ) being freshly prepared, it flowed 90 min until an adsorption plateau reached. And then, the chips were washed by the buffer to remove loosely attached protein 
molecules and reestablished the baseline. QCM-D experiments were repeated at least three times and average values were reported. The 3rd, 5th, 7th, and 9th overtones were used for all modeling calculations.

\section{Ellipsometry measurements}

The thickness of protein layers was also measured by a spectroscopic ellipsometer (M-2000V, J. A. Woollam) carrying out with a wavelength range from 370.1 to $999.1 \mathrm{~nm}$ at an incidence angle of $70^{\circ}$. The complex reflection coefficient is measured as a function of wavelength expressed by eqn (3): ${ }^{31}$

$$
\tan (\Psi) \mathrm{e}^{\mathrm{i} \Delta}=\frac{R_{\mathrm{p}}}{R_{\mathrm{s}}}
$$

where $\tan (\Psi)$ is the amplitude ratio of the reflection coefficient of p-polarized light $\left(R_{\mathrm{p}}\right)$ and that of s-polarized light $\left(R_{\mathrm{s}}\right) ; \Delta$ is the phase difference. The quantities of $\Psi$ and $\Delta$ can be obtained through an appropriate model. In this study, a well-established Cauchy dispersion model has been used to fit ellipsometry data, ${ }^{32,33}$ as described in detail in Fig. S1. $\dagger$ After the thickness being obtained (as shown in Table S1 $\dagger$ ), the adsorbed mass was calculated, where the density of the protein was chosen as $1.00 \mathrm{~g} \mathrm{~cm}^{-3} \cdot{ }^{34}$

The comparison between the adsorbed mass obtained from QCM-D, $m_{\mathrm{w}}$, and ellipsometry, $m_{\mathrm{d}}$, allows us to obtain the mass of water associated with layers using the method introduced previously. ${ }^{35,36}$ Relative water content (RWC), in the adsorbed film, can be calculated by eqn (4):
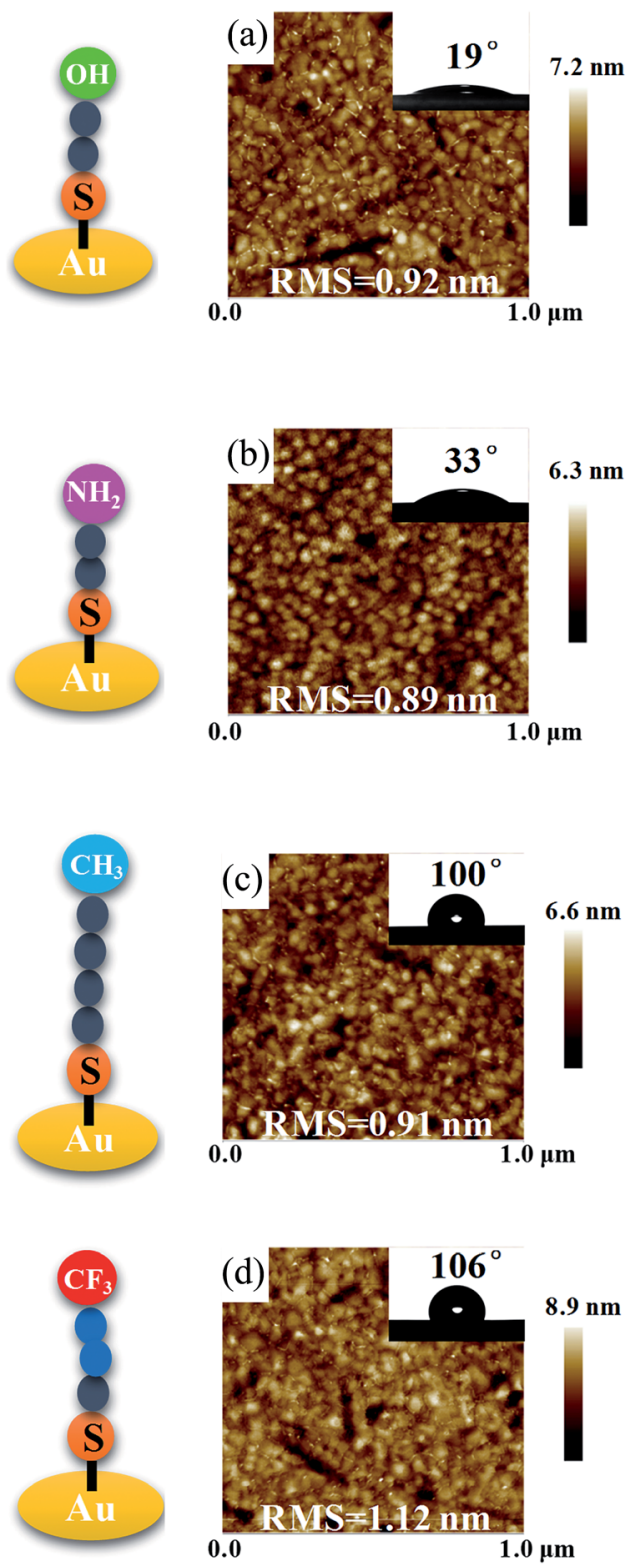
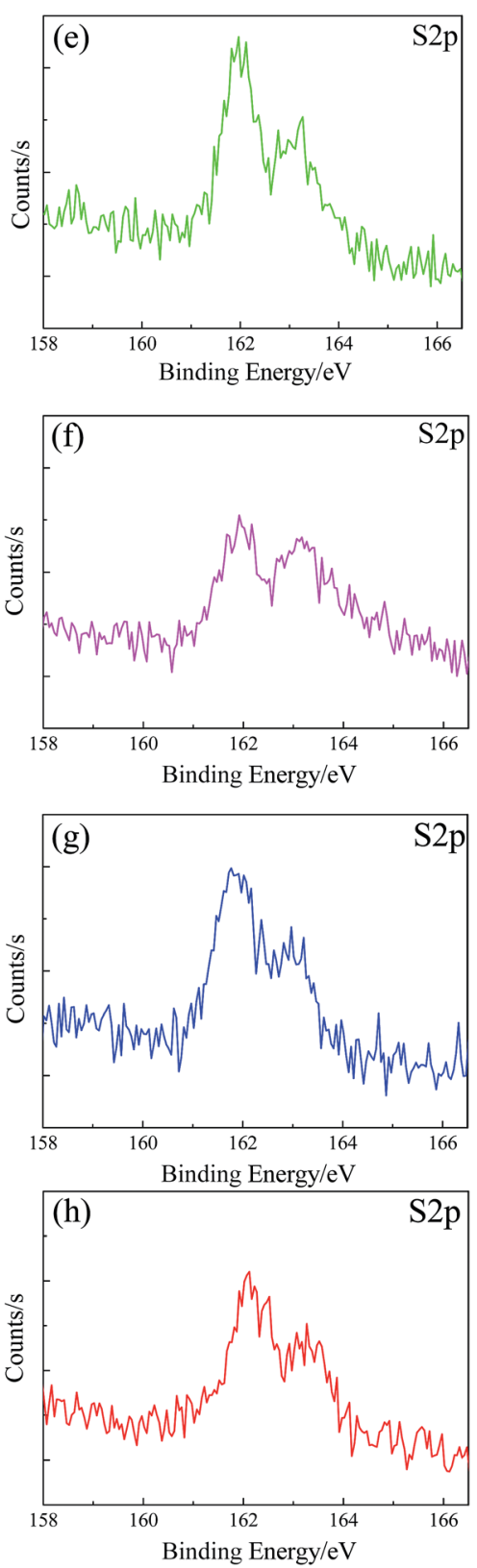

Fig. 1 Characterization of SAMs on gold: AFM images (a-d), water contact angle (inset figures), and XPS S2p spectra of $-\mathrm{OH},-\mathrm{NH}_{2},-\mathrm{CH}_{3}$, and $-\mathrm{CF}_{3}$ terminated surfaces $(\mathrm{e}-\mathrm{h})$. 


$$
\mathrm{RWC} \approx \frac{m_{\mathrm{w}}-m_{\mathrm{d}}}{m_{\mathrm{w}}}
$$

\section{Atomic force microscope (AFM)}

To investigate surface morphology of films obtained from QCMD measurements after equilibrium adsorption and rinsing, AFM images were recorded under air by a Fastscan mode (Nanoscope IIIa, Digital Instruments), with a pyramidal $\mathrm{SiO}_{2}$ tip $\left(4.0 \mathrm{~N} \mathrm{~m}^{-1}\right)$ and in a peakforce tapping mode. The root-meansquare (RMS) roughness was evaluated from the recorded AFM images and determined by using the Nanoscope Analysis software.

\section{Results and discussion}

\section{Surface characterization of SAMs}

By varying the end-group functionalities of thiols, we obtained a series of SAM-modified surfaces. From X-ray photoelectron spectroscopy (XPS) results (Fig. 1e-h), a peak at around $162 \mathrm{eV}$ is observed and attributed to the Au-S binding bond, indicating the successful modification of SAM end-groups (including SAM$\mathrm{OH}, \mathrm{SAM}-\mathrm{NH}_{2}, \mathrm{SAM}-\mathrm{CH}_{3}$, and SAM-CF ${ }_{3}$ ). The contact angle of water droplet on such surfaces increases from $\sim 19^{\circ}$ to $\sim 106^{\circ}$, implying that the model surfaces varying in water wettability are obtained. Nearly all these surfaces present a flat and homogenous conformation with similar average roughness (RMS) values of $\sim 1 \mathrm{~nm}$, as shown in Fig. 1a-d. Ellipsometric measurements also demonstrate that the film thickness of $-\mathrm{OH}$, $-\mathrm{NH}_{2},-\mathrm{CH}_{3}$, and $-\mathrm{CF}_{3}$ terminated surfaces is of about $1 \mathrm{~nm}$ (see Table $\mathrm{S} 1 \dagger)$.

\section{Mfp-1 adsorption}

Upon addition of Mfp-1 onto different kinds of surfaces, a rapid decrease in frequency $(\Delta f)$ and a slow increase in dissipation $(\Delta D)$ are observed as shown in Fig. 2. After the equilibrium adsorption, a rinse of pure buffer solution is introduced and some loosely attached proteins are removed. $\Delta f$ reaches at $\sim-47, \sim-56, \sim-64$, and $\sim-79 \mathrm{~Hz}$ in the case of Mfp-1 adsorbing onto the surfaces with $\mathrm{OH}, \mathrm{CH}_{3}, \mathrm{CF}_{3}$, and $\mathrm{NH}_{2}$ end groups, respectively. The frequency is accompanied by a similar dissipation value of about $1 \times 10^{-6}$. It indicates that the mass uptake of Mfp-1 on the hydrophilic modified surface (such as -OH terminated surface) may be lower than that on the bare surface as well as hydrophobic modified surface (such as $-\mathrm{CH}_{3}$ terminated and $-\mathrm{CF}_{3}$ terminated surfaces), except for the $-\mathrm{NH}_{2}$ terminated surface. At the testing $\mathrm{pH}(\sim 5.5)$, both Mfp-1 and $-\mathrm{NH}_{2}$ terminated surface are positively charged due to the protonation of the amine moiety, and thereby there is electrostatic repulsion between $\mathrm{Mfp}-1$ and the $-\mathrm{NH}_{2}$ terminated surface. The strongest adsorption on the $-\mathrm{NH}_{2}$ terminated surface suggests that there should be a strong attraction which can overcome the electrostatic repulsion. Recent SFA studies with Mfp-1 shows that long-range electrostatic repulsion in Mfp- 1 could be overcome by the short-range cation $-\pi$ attraction between amino groups on the surface and the aromatic groups

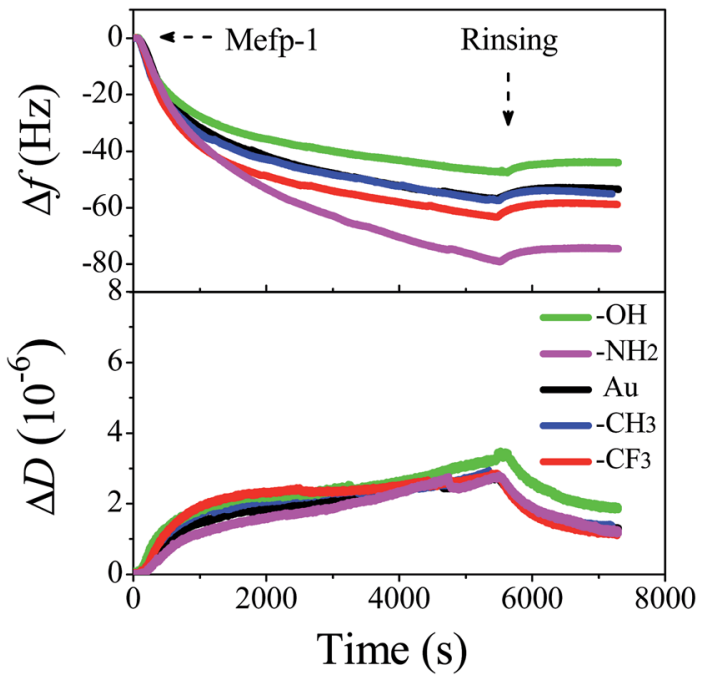

Fig. 2 Changes in frequency $(\Delta f)$ and dissipation $(\Delta D)$ as a function of time for the adsorption of Mfp-1 on the modified surfaces using QCM-D.

(tyrosine, DOPA, tryptophan, indole) of Mfp- $1 .^{37,38}$ The cation- $\pi$ interaction between positively charged amine group on the $-\mathrm{NH}_{2}$ terminated surface and DOPA/tyrosines in Mfp- 1 is likely one of the reasons of the strongest adsorption on the $-\mathrm{NH}_{2}$ terminated surface. It is speculated that there is a relationship between adsorbed mass and surface wettability, surface chemistry, as well as water load of the adsorbed protein layer, as described in the following. Information about mass and thickness of the adsorbed layer can be obtained through fitting values of $\Delta f$ and $\Delta D$ at different overtones by the use of Voigt model, as show in Fig. S2. $\dagger$

\section{Kinetic processes}

To investigate the adsorption kinetics of Mfp-1 on different surfaces, the profiles of $\Delta D v s$. $\Delta f$ are exhibited in Fig. 3. The values of slopes obtained by linear fit of the curves (see Fig. 3) for the first $\left(k_{1}\right)$ and second $\left(k_{2}\right)$ kinetic processes are shown in Table 1. During the adsorption process of protein, changes in the slope typically indicate that the proteins binding to the surface undergo structural rearrangements. ${ }^{39,40}$ At hydrophilic surfaces, dissipation per unit of $f$-shift is bigger in the second kinetic process, resulting in the bigger value of $k_{2}$ compared

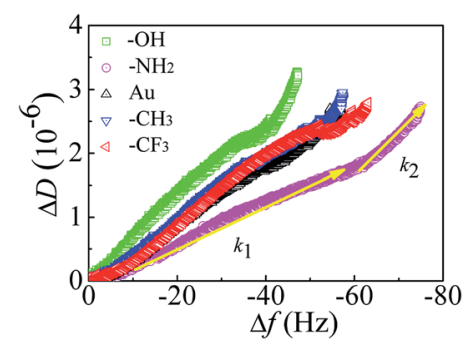

Fig. $3 \Delta D-\Delta f$ plots for the adsorption of Mfp-1 onto different substrates. 
Table 1 Values of $k_{1}$ and $k_{2}$ for the first and second kinetic processes, respectively

\begin{tabular}{lccccc}
\hline & \multicolumn{2}{l}{ Different surfaces } & & & \\
\cline { 2 - 6 } & $-\mathrm{OH}$ & $-\mathrm{NH}_{2}$ & $\mathrm{Au}$ & $-\mathrm{CH}_{3}$ & $-\mathrm{CF}_{3}$ \\
\hline$k_{1}\left(-10^{-8} / \mathrm{Hz}\right)$ & 6.17 & 3.05 & 5.11 & 5.68 & 5.39 \\
$k_{2}\left(-10^{-8} / \mathrm{Hz}\right)$ & 14.2 & 6.81 & 5.98 & 3.80 & 4.17 \\
\hline
\end{tabular}

with $k_{1}$ (see Table 1), and therefore the structural rearrangements may lead to a loose and soft adsorbed protein film during the continuous adsorption of protein. Especially in the case of -OH surface, the dissipation is the highest of all the surfaces and the value of $|f|$ is the smallest, so the value of $k_{2}$ is much higher than that of $k_{1}$, leading to accommodate a swelling conformation of the protein molecules and the weakest adsorption compared with other cases. In the case of hydrophobic surfaces, the first kinetic process with a similar value of $k_{1}$ corresponds to the initially fast adsorption of protein molecules onto the surfaces, and the second kinetic process with a lower value of $k_{2}$ relates to the structural rearrangements or conformational changes, resulting in a relatively rigid and dense protein layer.

Fig. 4 shows adsorbed mass of Mfp- 1 on modified surfaces calculated from QCM-D measurements using the Voigt model. In initial time, adsorption rate on SAM- $\mathrm{NH}_{2}$ surface is the highest among all the surfaces, probably because of the strong cation $-\pi$ interaction. After rinsing the adsorbed mass is in the increasing order: SAM-OH $<\mathrm{SAM}-\mathrm{CH}_{3}<\mathrm{SAM}-\mathrm{CF}_{3}<\mathrm{SAM}-\mathrm{NH}_{2}$. The results reveal that the adsorption rate and adsorbed mass of

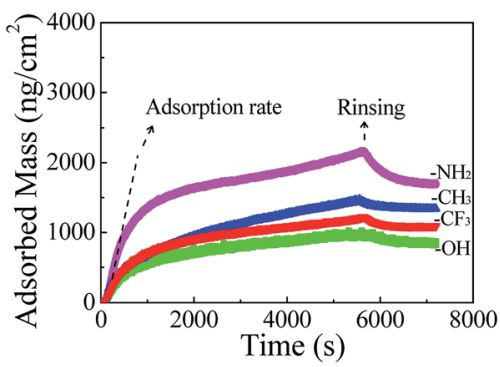

Fig. 4 Adsorbed mass of Mfp-1 on modified surfaces measured from QCM-D data using the Voigt model.
Mfp-1 on SAM-OH is the lowest among all the SAM substrates, mainly due to the hydration interactions between protein and the modified surface, which avoids the rapid adsorption of protein on the surface, resulting in low adsorbed quantity.

\section{QCM-D versus ellipsometry}

There is a substantial difference between the adsorbed masses calculated from QCM-D and ellipsometry measurements, because QCM-D mass includes both the mass of adsorbed protein and associated water, whereas ellipsometry mass gives a dry mass. QCM-D mass calculated through Voigt model is approximately double as compared to that of ellipsometry mass, showing a good ability in associating with water (Fig. 5). The water content decreases with the increase of hydrophobicity of the surface. On the relatively hydrophilic surfaces (e.g., $-\mathrm{OH}$ terminated surface), the adsorbed quantities are low and the water contents are high, resulting in the loose bond of protein molecules onto the surfaces. It suggests that the hydroxyl terminated thiol creates a hydration layer via hydrogen bonds between $-\mathrm{OH}$ groups and water molecules, forming a strong physical barrier to prevent direct contact between Mfp- 1 and the surface, ${ }^{41}$ which provides a further explanation on the low adsorption mass. On the contrary, hydrophobic surfaces exhibit low water contents (e.g., $-\mathrm{CH}_{3}$ or $-\mathrm{CF}_{3}$ terminated surface), owing to the weak barrier from Mfp-1 adsorption at surface and the formation of hydrophobic interactions between end groups and protein molecules.

\section{Surface characterization of protein films}

On the hydroxyl terminated surface, it is found that the protein preferentially forms a homogeneous layer with a low roughness of $\sim 1 \mathrm{~nm}$ (Fig. 6a). A high coverage of $\mathrm{Mfp}-1$ on the $-\mathrm{NH}_{2}$ terminated surface is obtained in according with the high adsorbed mass from QCM-D results, probably owing to the strong cation $-\pi$ interaction of protein and terminal amino of surface which is strong enough to overcome the electrostatic repulsion between them. ${ }^{40-42} \mathrm{Mfp}-1$ molecules tend to aggregate and form islands in larger size on the hydrophobic surface (including $-\mathrm{CH}_{3}$ terminated and $-\mathrm{CF}_{3}$ terminated surfaces), probably due to the hydrophobic interaction between the residue of DOPA and the hydrophobic groups on the SAMs. XPS C1s spectra results show that new peaks appear at $286.3 \mathrm{eV}$ and
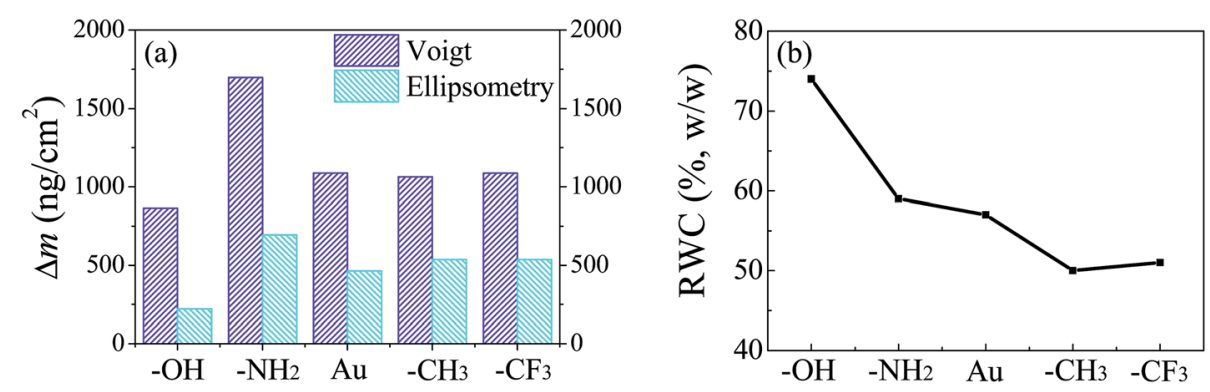

Fig. 5 (a) Adsorbed mass of Mfp-1 on different surfaces obtained from QCM-D and ellipsometry measurements; (b) relative water content (RWC) in the protein films estimated from QCM-D and ellipsometry mass. 
$288.1 \mathrm{eV}$, based on the $\mathrm{C}-\mathrm{O}$ or $\mathrm{C}-\mathrm{N}$ bond and amide bond ( $\mathrm{N}-$ $\mathrm{C}=\mathrm{O}$ ) from the protein comparing with the surface before protein absorption. On the $-\mathrm{OH}$ surface, the relative composition for the $\mathrm{N}-\mathrm{C}=\mathrm{O}$ peak at $288.1 \mathrm{eV}$ was $5.3 \%$, lower than that of the samples on other surfaces in accordance with the results from QCM-D and ellipsometry.
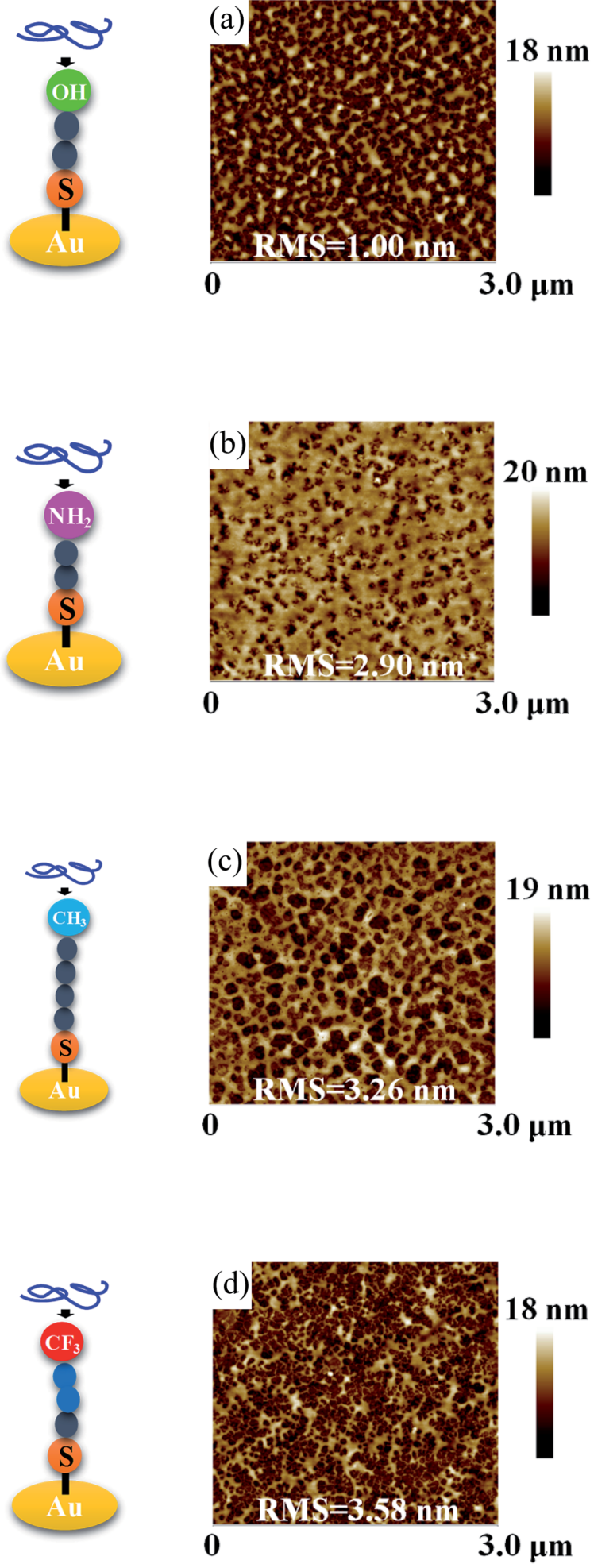
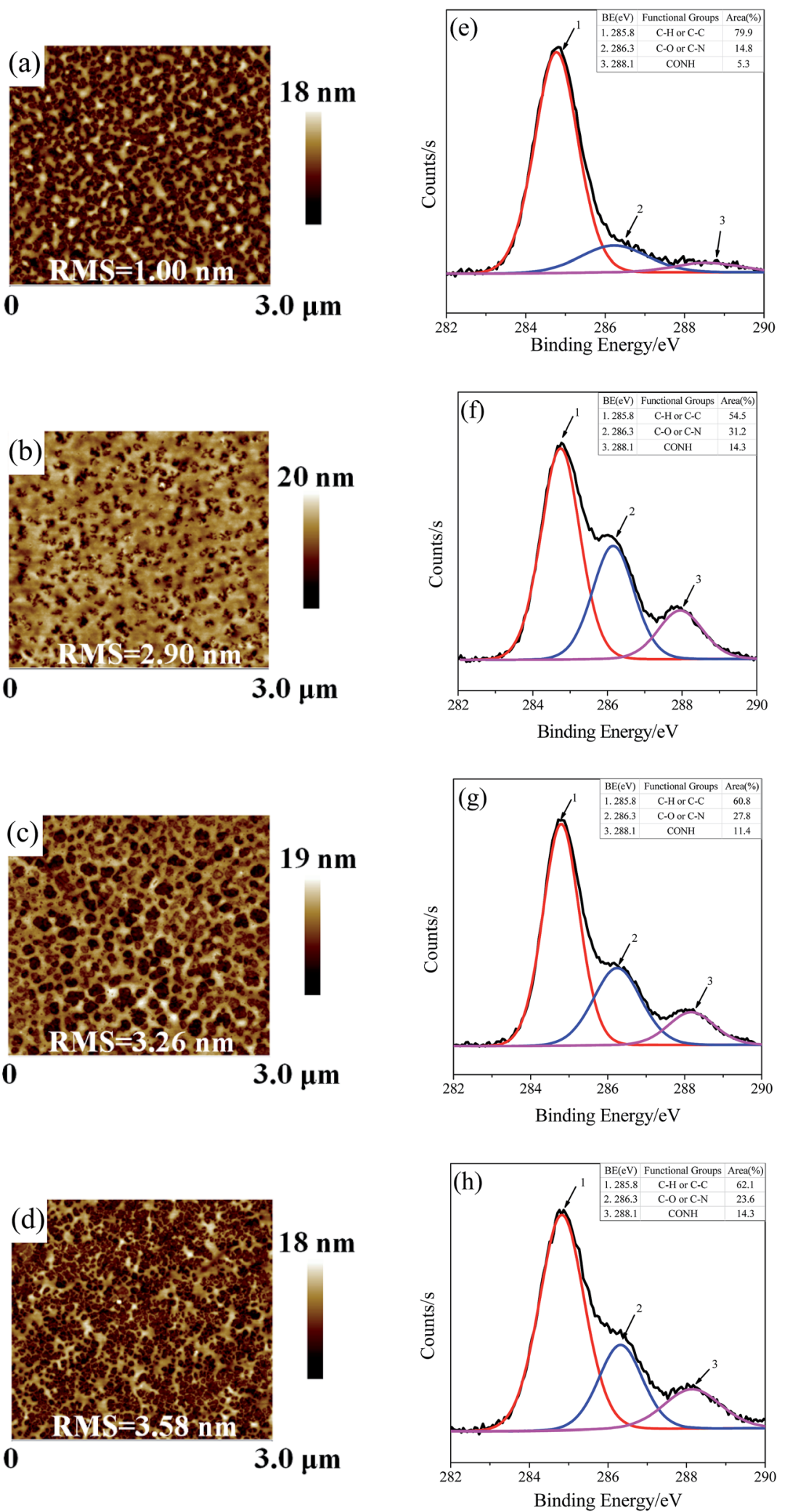

Fig. 6 AFM images and XPS C1s spectra of Mfp-1 on modified surfaces of SAM-OH (a, e), SAM-NH $(b, f), \mathrm{SAM}_{2} \mathrm{CH}_{3}(\mathrm{c}, \mathrm{g})$, and $\mathrm{SAM}-\mathrm{CF} \mathrm{F}_{3}(\mathrm{~d}, \mathrm{~h})$. 


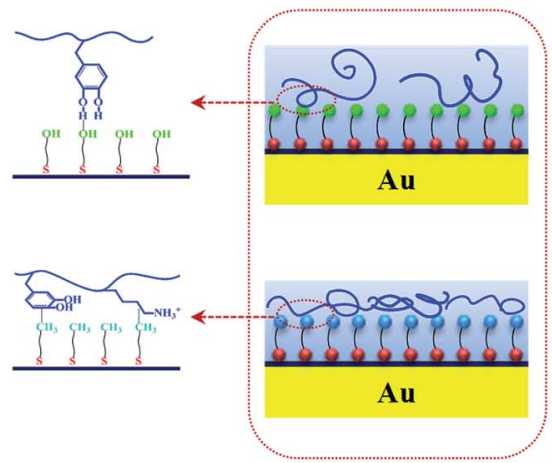

Kinetic process I

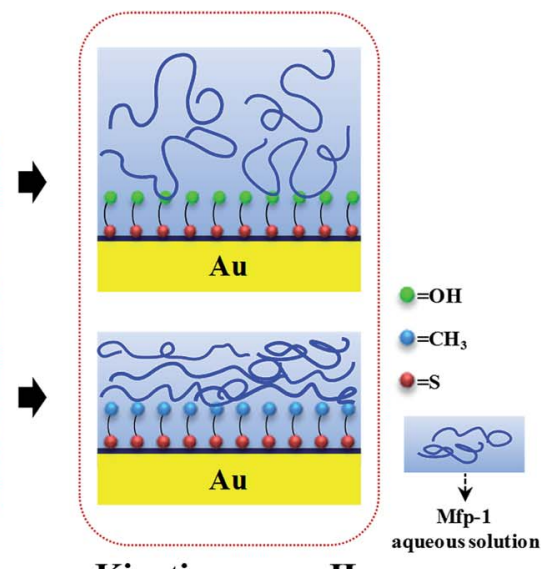

Kinetic process II

Fig. 7 A schematic of adhesion mechanisms of Mfp-1 on surfaces.

\section{Proposed adsorption mechanisms}

On the $-\mathrm{OH}$ terminated surface, hydrogen bonds can form between hydroxyl groups (hydrogen donors) of DOPA residues and the oxygen atoms (hydrogen acceptors) on the surface (see Fig. 7). And it has been confirmed that each DOPA presumably forms only one hydrogen bond with the OH-SAM headgroup. ${ }^{19}$ After protein molecules covering such surface, there is a structural rearrangement with the arrival of subsequent proteins. Due to the formation of loose and soft protein films with high water content, it can be concluded that water molecules tend to form hydrogen bonds with proteins and $-\mathrm{OH}$ terminated surface (so-called hydration layers), which plays an important role in preventing the adsorption of Mfp-1. Both amine terminated SAM and Mfp- 1 are positively charged in the experimental condition, but Mfp-1 shows the highest adsorption on the $-\mathrm{NH}_{2}$ terminated surface among all the SAM surfaces, probably due to the cation- $\pi$ interaction of protein-surface which is strong enough to overcome the electrostatic repulsion in wet conditions. ${ }^{42-45}$ On hydrophobic surface, taking $-\mathrm{CH}_{3}$ terminated surface as an example, DOPA is capable of interacting hydrophobically with the alkyl surface through its aromatic ring (Fig. 7). Other amino acid residues, containing a $\left(\mathrm{CH}_{2}\right)_{4}$ block, may improve the hydrophobic adhesion as well, ${ }^{46}$ and therefore, it shows that the surface is coated rigidly and densely. In addition, the shear viscosity and modulus values of Mfp-1 adsorbed at the hydrophilic OH-SAM surface are smaller than that of the hydrophobic $\mathrm{CH}_{3}$-SAM surface (Table $\mathrm{S} 2 \dagger$ ), consistent with a higher level of hydration at the OH-SAM surface. From all the results above, it suggests that subtle adjustments to surface chemistry can inhibit or enhance adhesion between Mfp-1 and tuning surfaces. This finding has important implications for shielding or improving the performance of environmental and medical coatings or adhesives on surfaces.

\section{Conclusion}

We have systematically studied the adsorption of Mfp-1 on the self-assembled monolayers (SAMs) varying from surface wettability and chemistry. The adsorption kinetics of the protein appeared to be distinct between such surfaces, which indicated the effect of surface wettability and chemistry on the restructure of Mfp-1 during adsorbing. Compared the mass of adsorbed protein on the surfaces measured by ellipsometry and quartz crystal microbalance with dissipation, it was found that the associated water played an essential role in preventing protein adsorption. At SAM-OH surface, the absorbed mass and adsorption rate of the protein were quite low probably due to the form of hydration layers on hydroxyl moieties. In the case of hydrophobic surface, such as $\mathrm{SAM}-\mathrm{CH}_{3}$ surface, it showed a high level of protein adsorption, because the adsorption process was dominated by the hydrophobic interactions of protein-surface and subsequently by the protein structural rearrangements. Besides, the adsorption behavior between the positively charged amine surface and positively charged Mfp-1 showed the strongest in QCM-D quantification among all the SAM surfaces because of the key interaction of cation- $\pi$ interaction between $\mathrm{NH}_{2}$-SAM and Mfp-1. Our results suggested that the adjustments to film chemistry was able to obtain either stronger or weaker adsorption of Mfp-1 onto model surfaces. It led a better understanding of the adsorption kinetics and structural conformation of protein on such surfaces, of great importance in developing medical materials or preventing marine fouling.

\section{Conflicts of interest}

The authors declare no competing financial interest.

\section{Acknowledgements}

This work was funded by the Foundation of PetroChina on Basic Research and Strategic Reserve Technology Research (Study on the regulation mechanisms of active controllable surfactants), the Important National Science and Technology Specific Project of China (2017ZX05013-003 and 2016ZX05025-003-009), and the National Natural Science Foundation of China (21603240). We 
thank Dr Min Wang from Biolin Scientific $\mathrm{AB}$ for the help in QCM measurements.

\section{References}

1 N. Holten-Andersen, H. Zhao and J. H. Waite, Stiff Coatings on Compliant Biofibers: The Cuticle of Mytilus Californianus Byssal Threads, Biochemistry, 2009, 48, 2752-2759.

2 D. J. Rubin, A. Miserez and J. H. Waite, Diverse Strategies of Protein Sclerotization in Marine Invertebrates: StructureProperty Relationships in Natural Biomaterials, Adv. Insect Physiol., 2010, 38, 75-133.

3 K. Inoue and S. Odo, The Adhesive Protein cDNA of Mytilus Galloprovincialis Encodes Decapeptide Repeats but No Hexapeptide Motif, Biol. Bull., 1994, 186, 349-355.

4 J. H. Waite and M. L. Tanzer, Polyphenolic Substance of Mytilus Edulis: Novel Adhesive Containing L-Dopa and Hydroxyproline, Science, 1981, 212, 1038-1040.

$5 \mathrm{~J} . \mathrm{Yu}$, Adhesive Interactions of Mussel Foot Proteins, Springer, 2014.

6 T. Williams, K. Marumo, J. H. Waite and R. W. Henkens, Mussel Glue Protein has an Open Conformation, Arch. Biochem. Biophys., 1989, 269, 415-422.

7 M. P. Deacon, S. S. Davis, J. H. Waite and S. E. Harding, Structure and Mucoadhesion of Mussel Glue Protein in Dilute Solution, Biochemistry, 1998, 37, 14108-14112.

8 D. S. Hwang and J. H. Waite, Three Intrinsically Unstructured Mussel Adhesive Proteins, Mfp-1, Mfp-2, and Mfp-3: Analysis by Circular Dichroism, Protein Sci., 2012, 21, 1689-1695.

9 D. B. Amabilino, Supramolecular Chemistry at Surfaces, Royal Society of Chemistry, 2016.

10 N. R. Martinez Rodriguez, S. Das, Y. Kaufman, J. N. Israelachvili and J. H. Waite, Interfacial pH during Mussel Adhesive Plaque Formation, Biofouling, 2015, 31, 221-227.

11 J. Yu, W. Wei, E. Danner, R. K. Ashley, J. N. Israelachvili and J. H. Waite, Mussel Protein Adhesion Depends on Interprotein Thiol-Mediated Redox Modulation, Nat. Chem. Biol., 2011, 7, 588-590.

12 S. Seo, S. Das, P. J. Zalicki, R. Mirshafian, C. D. Eisenbach, J. N. Israelachvili, J. H. Waite and B. K. Ahn, Microphase Behavior and Enhanced Wet-Cohesion of Synthetic Copolyampholytes Inspired by a Mussel Foot Protein, $J$. Am. Chem. Soc., 2015, 137, 9214-9217.

13 S. H. Yang, S. M. Kang, K. B. Lee, T. D. Chung, H. Lee and I. S. Choi, Mussel-Inspired Encapsulation and Functionalization of Individual Yeast Cells, J. Am. Chem. Soc., 2011, 133, 2795-2797.

14 B. D. Winslow, H. Shao, R. J. Stewart and P. A. Tresco, Biocompatibility of Adhesive Complex Coacervates Modeled after the Sandcastle Glue of Phragmatopoma Californica for Craniofacial Reconstruction, Biomaterials, 2010, 31, 9373-9381.

15 C. J. Kastrup, M. Nahrendorf, J. L. Figueiredo, H. Lee, S. Kambhampati, T. Lee, S. W. Cho, R. Gorbatov, Y. Iwamoto and T. T. Dang, Painting Blood Vessels and
Atherosclerotic Plaques with an Adhesive Drug Depot, Proc. Natl. Acad. Sci. U. S. A., 2012, 109, 21444-21449.

16 D. S. Hwang, M. J. Harrington, Q. Lu, A. Masic, H. Zeng and J. H. Waite, Mussel Foot Protein-1 (Mcfp-1) Interaction with Titania Surfaces, J. Mater. Chem., 2012, 22, 15530-15533.

17 J. Yu, W. Wei, E. Danner, J. N. Israelachvili and J. H. Waite, Effects of Interfacial Redox in Mussel Adhesive Protein Films on Mica, Adv. Mater., 2011, 23, 2362.

18 J. M. Curran, R. Chen and J. A. Hunt, The Guidance of Human Mesenchymal Stem Cell Differentiation in Vitro by Controlled Modifications to the Cell Substrate, Biomaterials, 2006, 27, 4783-4793.

19 J. Yu, Y. Kan, M. Rapp, E. Danner, W. Wei, S. Das, D. R. Miller, Y. Chen, J. H. Waite and J. N. Israelachvili, Adaptive hydrophobic and hydrophilic interactions of mussel foot proteins with organic thin films, Proc. Natl. Acad. Sci. U. S. A., 2013, 110, 15680-15685.

20 Y. Luan, D. Li, Y. Wang, X. Liu, J. L. Brash and H. Chen, 125Iradiolabeling, surface plasmon resonance, and quartz crystal microbalance with dissipation: three tools to compare protein adsorption on surfaces of different wettability, Langmuir, 2014, 30, 1029-1035.

21 Q. Lu, E. Danner, J. H. Waite, J. N. Israelachvili, H. Zeng and D. S. Hwang, Adhesion of Mussel Foot Proteins to Different Substrate Surfaces, J. R. Soc., Interface, 2013, 10, 20120759.

22 J. Yu, W. Wei, M. S. Menyo, A. Masic, J. H. Waite and J. N. Israelachvili, Adhesion of Mussel Foot Protein-3 to $\mathrm{TiO}_{2}$ Surfaces: The Effect of pH, Biomacromolecules, 2013, 14, 1072-1077.

23 Q. Lin, D. Gourdon, C. Sun, N. Holten-Andersen, T. H. Anderson, J. H. Waite and J. N. Israelachvili, Adhesion mechanisms of the mussel foot proteins mfp-1 and mfp-3, Proc. Natl. Acad. Sci. U. S. A., 2007, 104(10), 3782-3786.

24 Z. A. Levine, M. V. Rapp, W. Wei, R. G. Mullen, C. Wu, G. H. Zerze, J. Mittal, J. H. Waite, J. N. Israelachvili and J.-E. Shea, Surface force measurements and simulations of mussel-derived peptide adhesives on wet organic surfaces, Proc. Natl. Acad. Sci. U. S. A., 2016, 113, 4332.

25 R. B. Martin, Zwitterion Formation upton Deprotonation in L-3, 4-Dihydroxyphenylalanine and Other Phenolic Amines, J. Phys. Chem., 1971, 75, 2657-2661.

26 L. Pettit, Critical Survey of Formation Constants of Complexes of Histidine, Phenylalanine, Tyrosine, L-DOPA and Tryptophan, Pure Appl. Chem., 1984, 56, 247-292.

27 M. Mrksich, G. B. Sigal and G. M. Whitesides, SurfacePlasmon Resonance Permits in-Situ Measurement of Protein Adsorption on Self-Assembled Monolayers of Alkanethiolates on Gold, Langmuir, 1995, 11, 4383-4385.

28 G. Sauerbrey, Verwendung Von Schwingquarzen Zur Wägung DÜNner Schichten Und Zur Mikrowägung, $Z$. Phys., 1959, 155, 206-222.

29 L. Mivehi, R. Bordes and K. Holmberg, Adsorption of Cationic Gemini Surfactants at Solid Surfaces Studied by QCM-D and SPR: Effect of the Rigidity of the Spacer, Langmuir, 2011, 27, 7549-7557.

30 C. Laguerie, M. Aubry and J. P. Couderc, Some Physicochemical Data on Monohydrate Citric-Acid 
Solutions in Water - Solubility, Density, Viscosity, Diffusivity, pH of Standard Solution, and Refractive-Index, J. Chem. Eng. Data, 1976, 21, 85-87.

$31 \mathrm{H}$. Fujiwara, Spectroscopic ellipsometry: principles and applications, John Wiley \& Sons, 2007.

32 X. Chu, J. Yang, G. Liu and J. Zhao, Swelling Enhancement of Polyelectrolyte Brushes Induced By External Ions, Soft Matter, 2014, 10, 5568-5578.

33 H. Yang, H. Duan, X. Wu, M. Wang, T. Chen, F. Liu, S. Huang, W. Zhang, G. Chen and D. Yu, Self-Assembly Behavior of Ultrahighly Charged Amphiphilic Polyelectrolyte on Solid Surfaces, Langmuir, 2016, 32, 11485-11491.

34 X. Chu, J. Yang, G. Liu and J. Zhao, Swelling enhancement of polyelectrolyte brushes induced by external ions, Soft Matter, 2014, 10, 5568-5578.

35 E. Guzman, F. Ortega, N. Baghdadli, C. Cazeneuve, G. S. Luengo and R. G. Rubio, Adsorption of Conditioning Polymers on Solid Substrates with Different Charge Density, ACS Appl. Mater. Interfaces, 2011, 3, 3181-3188.

36 T. J. Halthur and U. M. Elofsson, Multilayers of Charged Polypeptides as Studied by in Situ Ellipsometry and Quartz Crystal Microbalance with Dissipation, Langmuir, 2004, 20, 1739-1745.

37 S. Kim, J. Huang, Y. Lee, S. Dutta, H. Y. Yoo, Y. M. Jung, Y. Jho, H. Zeng and D. S. Hwang, Complexation and coacervation of like-charged polyelectrolytes inspired by mussels, Proc. Natl. Acad. Sci. U. S. A., 2016, 113(7), E847E853.

38 D. S. Hwang, H. Zeng, Q. Lu, J. Israelachvili and J. H. Waite, Adhesion mechanism in a DOPA-deficient foot protein from green mussels, Soft Matter, 2012, 8(20), 5640-5648.
39 F. Hook, B. Kasemo, T. Nylander, C. Fant, K. Sott and H. Elwing, Variations in Coupled Water, Viscoelastic Properties, and Film Thickness of a Mefp-1 Protein Film During Adsorption and Cross-Linking: A Quartz Crystal Microbalance with Dissipation Monitoring, Ellipsometry, and Surface Plasmon Resonance Study, Anal. Chem., 2001, 73, 5796-5804.

40 D. E. Otzen, M. Oliveberg and F. Hook, Adsorption of a Small Protein to a Methyl-Terminated Hydrophobic Surfaces: Effect of Protein-Folding Thermodynamics and Kinetics, Colloids Surf., B, 2003, 29, 67-73.

41 S. Chen, J. Zheng, L. Li and S. Jiang, Strong Resistance of Phosphorylcholine Self-Assembled Monolayers to Protein Adsorption: Insights into Nonfouling Properties of Zwitterionic Materials, J. Am. Chem. Soc., 2005, 127, 1447314478.

42 D. A. Dougherty, Cation- $\pi$ interactions in chemistry and biology: a new view of benzene, Phe, Tyr, and Trp, Science, 1996, 271(5246), 163.

43 J. C. Ma and D. A. Dougherty, The cation- $\pi$ interaction, Chem. Rev., 1997, 97(5), 1303-1324.

44 J. P. Gallivan and D. A. Dougherty, A computational study of cation $-\pi$ interactions vs. salt bridges in aqueous media: implications for protein engineering, J. Am. Chem. Soc., 2000, 122, 870-874.

45 S. Kim, A. Faghihnejad, Y. Lee, Y. Jho, H. Zeng and D. S. Hwang, Cation- $\pi$ interaction in DOPA-deficient mussel adhesive protein mfp-1, J. Mater. Chem. B, 2015, 3, 738-743.

46 T. E. Creighton, Proteins: structures and molecular properties, Macmillan, 1993. 